\title{
Molecularly imprinted potentiometric sensor for surfactant based on electrosynthesized polyaniline
}

\author{
Francis G. Biñas ${ }^{1}$ E Fortunato Sevilla III ${ }^{1,2}$ \\ ${ }^{1}$ College of Science; ${ }^{2}$ Research Center for the Natural and Applied Sciences \\ University of Santo Tomas, España Boulevard, 1015 Manila, PHILIPPINES
}

\begin{abstract}
A potentiometric sensor for sodium dodecylsulfate (SDS) was developed based on molecularly-imprinted polyaniline. The molecularly-imprinted polymer was synthesized through a potentiostatic electropolymerization of aniline monomer in the presence of the template SDS molecule at a constant potential of $0.8 \mathrm{~V}$. The polymerization parameters determined the analytical performance of the polyaniline reagent phase. The optimized electropolymerization process provided a sensor with sensitivity of $45.54 \pm 6 \mathrm{mV} /$ decade, working concentration range of $3 \times 10^{-6} \mathrm{M}$ to $1 \times 10^{-2} \mathrm{M}$ SDS, correlation coefficient $\left(r^{2}\right)$ of 0.993 and with a limit of detection $1 \times 10^{-5} \mathrm{M} \mathrm{DS}^{-}$. The sensor response is reproducible with a mean RSD of $8.08 \%$ from $3 \times 10^{-6} \mathrm{M}$ to $3 \times 10^{-3} \mathrm{M}$ SDS. Selectivity studies using the fixed primary ion method proved the higher selectivity of the synthesized polymer for SDS over inorganic salts and fatty acid salts.
\end{abstract}

Keywords: molecularly imprinted polyaniline, potentiometric sensor, sodium dodecysulfate

\section{INTRODUCTION}

Surfactants are widely used in detergents and other cleaning products. Large quantities of these products are consumed daily in households and industry, and are discharged into the aquatic environment through wastewater effluents [1]. The wide-spread usage has caused elevated levels of surfactants in the aquatic ecosystem where their toxic effects can impact on organisms in the water environment [2]. The amount of surfactant in the environment has to be monitored to ensure that toxic levels are not reached or surpassed.

\footnotetext{
*To whom correspondence should be addressed x1fractals@hotmail.com; fbsevilla@mnl.ust.edu.ph
}

The most common surfactants are the sodium alkylsulfates, such as SDS. The standard method for the determination of these surfactants is an extraction-spectrophotometric method known as the methylene blue active substances (MBAS) method [3]. A variety of instrumental methods have been applied for the measurement of these surfactants, including spectrophotometric, potentiometric and chromatographic techniques [4]. Most of these methods involve long analysis time, tedious sample preparation, expensive instrumentation and toxic solvents. Thus, there has been great interest in the development of simple, rapid and inexpensive methods for the measurement of surfactants, such as SDS. 
Chemical sensors present an attractive alternative for the quantitation of surfactants, providing simple and rapid measurements which can be done on-site and in real-time. These devices generate electrical responses which are related to the analyte concentration. Several transduction principles have been exploited in the development of chemical sensors for surfactants. Piezoelectric sensors for surfactants have been developed based on the adsorption of surfactant ion-pairs [5] and the binding of the surfactant with a polymer reagent phase [6]. An amperometric sensor has been fabricated which employed surfactant-degrading microorganisms [7]. Several potentiometric sensors had been developed employing ionselective PVC membranes [8-11] and conducting polymers $[12,13]$.

In this paper, a potentiometric sensor for sodium dodecylsulfate based on molecularly-imprinted polyaniline is described. A molecularly imprinted polymer contains microcavities bearing the shape of the template molecule, enabling it to bind selectively with this molecule. This feature imparts selectivity to the polymer, making it a good recognition element for sensors. Molecularly imprinted polyaniline has been applied in conductimetric sensors [14], several amperometric sensors [15], but to our knowledge, it has not been employed in potentiometric sensors, particularly to surfactants.

\section{EXPERIMENTAL}

Materials. All reagents employed in the fabrication of the sensor were analytical grade chemicals. Sodium dodecylsulfate (Sigma Chemicals) and graphite (Fluka) were used as received. Aniline monomer (Fluka) was distilled prior to its use as a starting material for the electro-polymerization. Buffer solutions were prepared using analytical grade reagents and distilled water: acetic acid (Merck), phosphoric acid (Ajax), boric acid (J.T. Baker), Tris (Fluka), sodium hydroxide (Ajax) and hydrochloric acid
(J. T. Baker). Epoxy resin (Epoteck H77) and hardener (Epotek H77B) were purchased from Epoxy Technology, U.S.A.

Fabrication of sensor. The sensor was constructed from an acrylic tube body provided with a copper disk and socket connector for electrical contacts, as described previously by Martinez and Alegret [16]. A conducting paste consisting of $0.5 \mathrm{~g}$ graphite powder and $0.5 \mathrm{~g}$ epoxy resin/hardener mixture, was applied on the copper disk at one end of the tube. The paste was allowed to cure overnight at $40^{\circ} \mathrm{C}$. The surface of the composite was polished to produce an even flat surface.

Polyaniline was deposited on the surface of the carbon/epoxy composite through potentiostatic electropolymerization at room temperature using a three-electrode cell. A silver-silver electrode (Crison 52-40) was used as the reference electrode, a platinum electrode (Crison 52-67) as the counter electrode, and the graphite/epoxy sensor as the working electrode. The three electrodes were connected to a potentiostat (BAS Petit Ampere LC-3E). A digital multimeter (Radioshack) was used for electrochemical measurement.

The polymerization mixture contained sodium dodecylsufate $(0.20 \mathrm{M})$ and aniline monomer $(0.10 \mathrm{M})$ dissolved in an aqueous buffer solution. The mixture was contained in a beaker, sealed with parafilm and protected from light by covering with aluminum foil. A constant potential of $+0.8 \mathrm{~V}$ was applied on the polymerization for $30 \mathrm{~min}$. After electrodeposition, the resulting polymer layer was washed to remove excess template and monomer, and then it was allowed to dry in atmospheric air.

Surface morphology study for both imprinted and non-imprinted polymers was conducted using Scanning Electron Microscope (SEM) model JSM-5310-JEOL. 
The following eletropolymerization parameters were optimized: SDS concentration, SDS:aniline ratio, $\mathrm{pH}$, electropolymerization time and applied potential.

Measurement procedure. Potentiometric measurements were carried out by immersing the sensor and a $\mathrm{Ag} / \mathrm{AgCl}$ reference electrode (Metrohm) in the test solution. The two electrodes were connected to a digital multimeter (Radioshack), which was interfaced to a computer. The sensor response was monitored until the voltage reading stabilizes for at least $\pm 0.1 \mathrm{mV} / \mathrm{min}$. The solution was continuously stirred during the measurement.

The following parameters were optimized: sensor conditioning time, sensor conditioning medium, $\mathrm{pH}$ and ionic strength of the test solution.

\section{Results AND DISCUSSION}

\section{Electrodeposition of molecularly-imprinted}

polyaniline. Molecularly imprinted polyaniline (MIP) was produced by applying a voltage through a solution containing the aniline monomer and SDS template molecules. Electrooxidation of the aniline monomer occurred at the anode, and the product polyaniline deposited on the surface of the carbon/epoxy composite electrode. A layer of the deposited polyaniline could bind SDS molecules through hydrogen bonding and electrostatic interactions, leading to the entrapment of template molecules in the polymer matrix as the electrooxidation proceeds. Branching and cross-linking reactions could occur [17] and lead to the formation of a threedimensional matrix containing entrapped SDS molecules. Washing with water removes the entrapped template molecule, leaving cavities bearing the shape of the template molecule.

The surface morphology of the synthesized polymers revealed by the SEM micrograph in Fig. 1A featured aggregations of small globular structures in the MIP which could be due to the molecularly-imprinted cavities in the polymer matrix. These aggregates were not observed in the SEM micrograph of the non-molecularlyimprinted polymer (Fig. 1B).

The recognition capability of the MIP is indicated by the greater sensitivity of the sensor containing the MIP compared to the sensor coated with non-imprinted polyaniline (NIP), as shown in the calibration curves in Fig. 2. The NIP generated electrical response to the SDS analyte due to non-specific adsorption on the polymer surface.
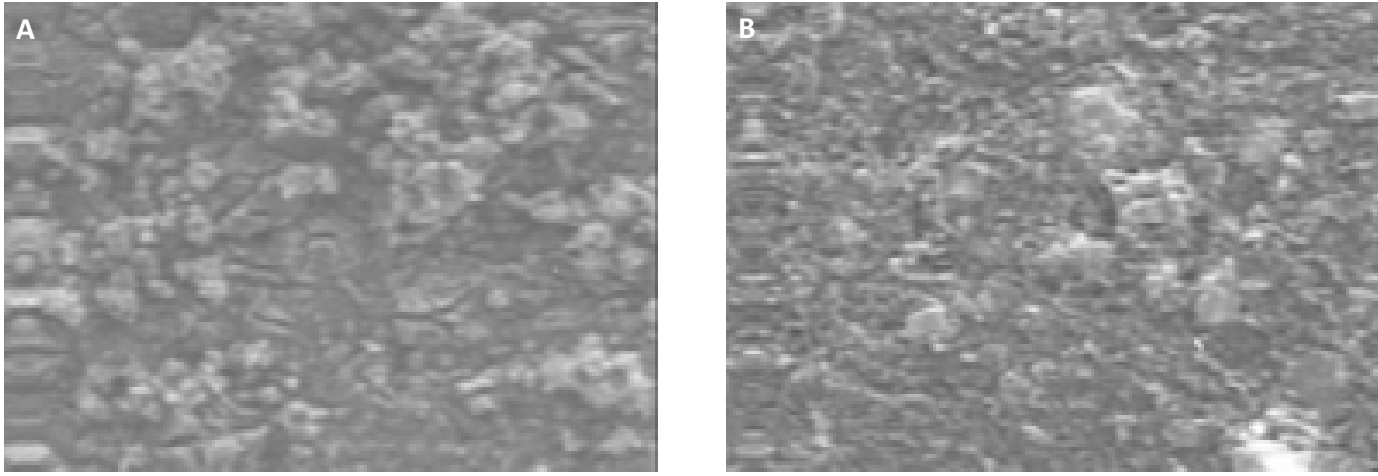

Figure 1. SEM micrographs of (A) SDS-molecularly-imprinted polyaniline, and (B) non-molecularly-imprinted polyaniline (Magnification: $750 \times$ ). 


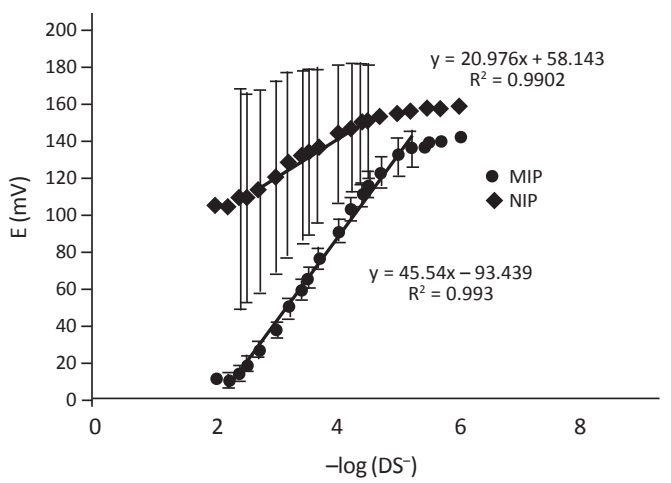

Figure 2. Calibration curves from sensors with MIP and NIP [Electropolymerization time: $30 \mathrm{~min}$, applied voltage:0.8 V; pH 5 acetate buffer, Conditioning time: $1 \mathrm{~h} ; n=5$ ]

The parameters employed in the electrosynthesis of the MIP influenced the analytical performance of the sensor. The applied potential, polymerization time, $\mathrm{pH}$ and SDS concentration affected the sensitivity and the linear range of the sensor.

The voltage applied during electropolymerization determined the properties of the MIP deposited on the anode surface. An applied potential of $0.8 \mathrm{~V}$ yielded a sensor with a near-Nernstian sensitivity and a wide linear range covering three orders of magnitude. The sensors produced by an applied potential of $0.5 \mathrm{~V}$ and $1.0 \mathrm{~V}$ had thin MIP membranes with low sensitivity, narrower linear range and poor stability. At an applied voltage of $0.5 \mathrm{~V}$, minimal polymer growth occurs [18], while at $1.0 \mathrm{~V}$, degradation of polyaniline takes place [19].

The length of the electropolymerization period affected the analytical sensitivity of the MIP sensing membrane. It had minimal effect on the linear range of the polymer. The highest sensitivity was observed from the membrane obtained at a polymerization time of $30 \mathrm{~min}$. A longer polymerization time yielded a thick film which showed an erratic behavior and a subNernstian sensitivity. The MIP obtained from a polymerization time of 5 min was thin and with a very poor sensitivity due to the small number of recognition sites existing in the polymer matrix.

The analytical performance of the electrochemically synthesized MIP was dependent on the $\mathrm{pH}$ of the polymerization solution. Electropolymerization of the aniline monomer at $\mathrm{pH} 5$ resulted in a membrane with the highest sensitivity, the highest magnitude $(\Delta \mathrm{E})$ of potentiometric reading and the wide linear range. The MIP formed at $\mathrm{pH}$ lower and higher than 5 had very poor sensitivity. At very low $\mathrm{pH}$, SDS exists in the molecular form and the aniline monomer is in the protonated form. As a result, very limited interaction takes place between the template and monomer molecules, and the formation of the recognition sites is not favored. At higher $\mathrm{pH}$, the polyaniline formed exhibits low conductivity [20] and therefore poor potentiometric behavior.

The concentration of the SDS in the electropolymerization solution affected the potentiometric characteristics of the MIP sensing membrane. The polymer produced from $0.20 \mathrm{M}$ exhibited a near-Nernstian sensitivity and a wide linear range. The membranes obtained at lower SDS concentrations showed subNernstian behavior and narrower linear range. These properties could be due to a smaller number of recognition sites formed in the MIP matrix. On the other hand, higher SDS concentrations also led to thicker sensing membranes with low sensitivity to SDS. The excess SDS molecules formed micelles with the monomer molecules, making them less available for binding in the growing polymer chain [21] and thus decreasing the number of binding sites.

Optimum performance was obtained from the MIP produced using the following electropolymerization parameters: concentration ratio aniline and SDS (1:2), medium ( $\mathrm{pH} 5$ acetate buffer), 30 min electropolymerization time, applied potential of $0.8 \mathrm{~V}$. 


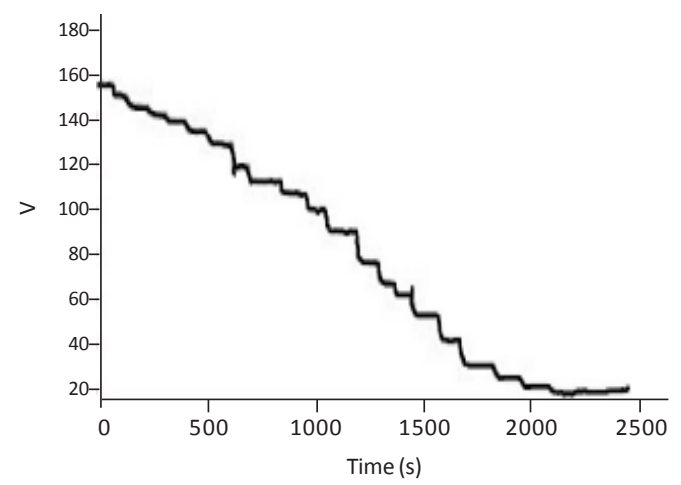

Figure 3. Potentiometric graph of PAni-SDS sensor

Potentiometric behavior. The sensor responded immediately to the presence of SDS, decreasing abruptly within a second and attaining a steady value within $20 \mathrm{~s}$. This behavior was exhibited after each addition of SDS standard solution to $25 \mathrm{~mL}$ distilled water (Fig. 3). As more SDS was added, the sensor required a shorter time to attain a steady state value.

The sensor response was reversible and reproducible. When the sensor was alternately immersed in $1 \times 10^{-3} \mathrm{M}$ and $1 \times 10^{-4} \mathrm{M}$ SDS standard solutions, it responded rapidly to the change in concentration. Its response to the two solutions exhibited good precision - the relative standard deviation was $3.99 \%$ for $1 \times 10^{-3} \mathrm{M}$ SDS and $3.70 \%$ for $1 \times 10^{-4} \mathrm{M}$ SDS.

The response of the sensor increased as the SDS concentration increased. A highly linear relationship was found to exist between the measured potential and the -log of SDS concentration, as shown by the high value of Pearson correlation coefficient (0.993) and discernible linear trend in Fig. 4. The linear behavior covered four order of magnitudes of SDS concentrations, ranging from $3 \times 10^{-6} \mathrm{M}$ to $1 \times 10^{-2} \mathrm{M}$. The sensitivity was near-Nernstian (45.54 mV/decade). The limit of detection was calculated as $1 \times 10^{-5} \mathrm{M}$ SDS.

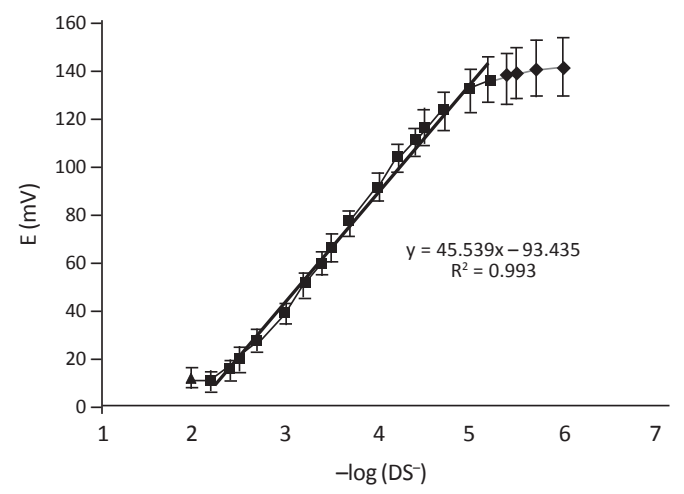

Figure 4. Calibration curve obtained from standard addition method using the PAni-SDS sensor.

The sensor responded to SDS more selectively than to several inorganic sodium salts, as shown by the selectivity coefficients in Table 1 . The selectivity coefficients were evaluated using the fixed primary ion method [22]. The selectivity towards SDS was also good in the presence of the sodium salt of long chain fatty acids, such as sodium laureate and sodium oleate (Table 1). This behavior confirms the molecular imprinting of the polypyrrole reagent phase. However, sodium dodecylbenzylsulfonate exhibited significant interference in the response of the SDS sensor. This interference could be due to the possibility of the interaction of the dodecylbenzenesulfonate ion with the protonated nitrogen atoms in the polymer phase.

The sensitivity of the PAni-SDS sensor was found to decrease by comparing the calibration curve before and after 31 days. During this

Table 1. Selectivity coefficients obtained for the potentiometric SDS sensor.

\begin{tabular}{l|c}
\hline \multicolumn{1}{c|}{ Interferents } & $\mathbf{K}_{\mathbf{A . B}}{ }^{\text {pot }}$ \\
\hline $\mathrm{Na}_{2} \mathrm{C}_{2} \mathrm{O}_{4}$ & 0.2 \\
\hline $\mathrm{Na}_{2} \mathrm{SO}_{4}$ & 0.2 \\
\hline $\mathrm{NaOAC}$ & 0.32 \\
\hline $\mathrm{NaBr}$ & 0.16 \\
\hline $\mathrm{NaCl}$ & 0.05 \\
\hline $\mathrm{SDBS}$ & 1.6 \\
\hline $\mathrm{C}_{17} \mathrm{H}_{33} \mathrm{COONa}$ & 0.32 \\
\hline $\mathrm{CH}_{3}\left(\mathrm{CH}_{2}\right)_{10} \mathrm{COONa}$ & 0.063 \\
\hline
\end{tabular}


period, the sensitivity deteriorated from $45.45 \mathrm{mv} /$ decade for the first day to $36.23 \mathrm{mV} /$ decade, after 30 days. The observed decrease in the sensor's performance with time may be an effect of external environmental agent, such as oxygen [4] and possible fouling of electrode every after use.

\section{CONCLUSION}

A potentiometric sensor for SDS was developed based on a molecularly imprinted polyaniline film deposited on a graphite rod. The sensor response exhibited good reproducibility and reversibility. It behaved linearly within the concentration rage of $3 \times 10^{-6} \mathrm{M}$ to $1 \times 10^{-2} \mathrm{M}$ SDS, and its limit of detection of about $1 \times 10^{-5} \mathrm{M}$ SDS. The ions from inorganic salts and fatty acid salts did not interfere with SDS.

\section{REFERENCES}

[1] Ivankovic T, Hrenovic J. Surfactants in the environment. Archives of Industrial Hygiene and Toxicology 2010; 61(1):95-110.

[2] Ying G. Fate, behavior and effects of surfactants and their degradation products in the environment. Environment International 2006; 32:417-531.

[3] American Public Health Association, American Water Works Association. Standard methods for examination of water and wastewater $\left(20^{\text {th }}\right.$ Edition). (American Public Health Association, 1998).

[4] Kulapina EG, Chernova RK, Markova NM, Pogorelova ES. Methods for determining synthetic surfactants. Review Journal of Chemistry 2013; 3:323-326.

[5] Nomura T, Egawa T. Adsorption determination of ionic surfactants using an electrode-separated piezoelectric quartz crystal. Analytica Chimica Acta 1997; 339:187-192.

[6] Albano DR, Sevilla III F. Piezoelectric quartz crystal sensor for surfactant based on molecularly imprinted polypyrrole. Sensors and Actuators $B$ 2007; 121:129-134.

[7] Taranova L, Semenchuk I, Manolov T, Iliasov P, Reshetilov A. Bacteria-degraders as the Base of an Amperometric Biosensor for Detection of Anionic Surfactants. Biosensors and Bioelectronics 2002; 17:635-640.
[8] Sànchez J, Beltran A, Alonso J, Jiménez C, Valle MD. Development of a new ion-selective fieldeffect transistor sensor for anionic surfactants: application to potentiometric titrations. Analytica Chimica Acta 1999; 382:157-164.

[9] Madunic-Cacica D, Sak-Bosnar M, Galovic O, Sakac N, Matesic-Puac R. Determination of cationic surfactants in pharmaceutical disinfectants using a new sensitive potentiometric sensor. Talanta 2008; 76:259-264.

[10] Najafi M, Maleki L, Abbas Rafati A. Novel surfactant selective electrochemical sensors based on single walled carbon nanotubes. Journal of Molecular Liquids 2011; 159:226-229.

[11] Wang J, Du Z, Wang W, Xue W. Ion-selective electrode for anionic surfactants using hexadecyl trimethyl ammonium bromide-sodium dodecylsulfate as an active ionophore. International Journal of Electrochemistry 2011; Article 958647 (7 pp.) (DOI:10.4061/2011/ 958647).

[12] Alizadeh N, Mahmodian M. A new dodecylsulfate ion-selective electrode based on electrochemically prepared polypyrrole and PVC. Electroanalysis 2000; 12:509-512.

[13] Mousavi MF, Shamsipur M, Riahi S, Rahmanifar MS. Design of a new dodecylsulfate selective electrode based on conductive polyaniline. Analytical Sciences 2002; 18:137-140.

[14] Musavi SM, Akbarzadeh A, Hosseini SH. Preparation of the Sensor of Imprinting Molecular Polymer Based on Polyaniline to Recognize Agricultural Toxin Chlorpyrifos and Diazinon. Life Science Journal (Acta Zhengzhou University Overseas Edition) 2012; 9(2):1280-1285.

[15] Zhao L, Du JY. Fabrication of chloramphenicol molecular imprinted composite film and its electrochemistry. Chinese Journal of Applied Chemistry 2012; 29:1212-1217.

[16] Martinez-Fabregas E, Alegret A. The The Modern Student Laboratory: A Practical Approach to Chemical Sensors through Potentiometric Transducers: Determination of Urea in Serum by Means of a Biosensor. Journal of Chemical Education 1994; 71:A67-70.

[17] Ciric-Marjanovic G. Recent advances in polyaniline research: Polymerization mechanisms, structural aspects, properties and applications. Synthetic Metals 2013; 177:1-47.

[18] Arsov LD. Electrochemical study of polyaniline deposited on a titanium surface. Journal of Solid State Electrochemistry 1988; 2:266-272. 
[19] Mazeikiene R, Malinauskas M. Kinetic study of the electrochemical degradation of polyaniline. Synthetic Metals 2001; 123:349-354.

[20] Kanungo M, Kumar A, Contractor AQ. Studies on electropolymerization of aniline in the presence of sodium dodecyl sulfate and its application in sensing urea. Journal of Electroanalytical Chemistry 2001; 528:46-56.
[21] Ping Z, Nauer GE, Neugebauer H, Theiner J, Neckel A. Protonation and electrochemical redox doping processes of polyaniline in aqueous solutions: Investigations using in-situ FTIR-ATR spectroscopy and a new doping system. Journal of the Chemical Society (Faraday Transactions) 1997; 93:121-129.

[22] Umezawa Y, Buhlmann P, Umezawa K, Tohda K, Amemiya A. Potentiometric Selectivity Coefficients of Ion-Selective Electrodes. Part I. Inorganic Cation. Pure and Applied Chemistry 2000; 72(10):1851-2082. 